\title{
Survey on the Use of Information and Communication Technology Tools in Educating Caregivers of Elderly People with Dementia
}

\section{-Analysis from Ichushi and PubMed}

\author{
Miwa Yamamoto1 ${ }^{*}$, Noriko Adachi ${ }^{1}$, Yasuko Maekawa ${ }^{2}$, Tomoharu Nakashima ${ }^{2}$, \\ Shigeru Sakuraba ${ }^{3}$ \\ ${ }^{1}$ Tottori University, Yonago, Japan \\ ${ }^{2}$ Osaka Prefecture University, Osaka, Japan \\ ${ }^{3}$ Kyoto Koka Women's University, Kyoto, Japan \\ Email: tokiwa27@hotmail.com
}

Received 29 December 2013; revised 18 March 2014; accepted 18 April 2014

Copyright (C) 2014 by authors and Scientific Research Publishing Inc.

This work is licensed under the Creative Commons Attribution International License (CC BY). http://creativecommons.org/licenses/by/4.0/

(c) (i) Open Access

\section{Abstract}

This study aimed to clarify the use of computer simulations in educating caregivers of elderly people with dementia. Thesaurus words in articles indexed in Ichushi and PubMed were analyzed using commercially available "Trend Search" text-mining software developed by FUJITSU. The analysis maps relational words in the articles, with line size and distance between words showing the strength of the relation. For ethical purposes, articles were anonymized for analysis. The search was conducted using the query phrases "elderly people with dementia AND simulation", and retrieved four articles from Ichushi (2007-2010) and 16 from PubMed (1992-2012). The search results revealed that little research has been done on this subject, and highlighted opportunities to further investigate the use of ICT tools in educating caregivers of elderly people with dementia.

\section{Keywords}

Survey, Elderly People with Dementia, ICT Tools

\section{Introduction}

The causes of dementia have not yet been clarified, and no effective treatment or cure is currently available. *Corresponding author.

How to cite this paper: Yamamoto, M., Adachi, N., Maekawa, Y., Nakashima, T. and Sakuraba, S. (2014) Survey on the Use of Information and Communication Technology Tools in Educating Caregivers of Elderly People with Dementia. Open Journal of Nursing, 4, 375-378. http://dx.doi.org/10.4236/ojn.2014.45043 
Furthermore, the need for healthcare research on elderly people with dementia is changing. The situation surrounding the elderly is being exacerbated in Japan, making it difficult to care for elderly people with dementia in hospitals. At the same time, greater societal awareness of the plight of elderly people with dementia has increased emphasis on delivering high-quality, efficient care to these patients. Thus, there is a need to invest in the education of people who care for these patients.

Information and Communication Technology (ICT) tools are one of the most effective means of education. Among the various ICT techniques that exist, computer simulation imitates the operation of a real-world process or system in real time. The simulation of an object or event first requires development of a model that represents the key characteristics or behaviors of the selected physical or abstract system or process. Such simulations have been used in various contexts, such as performance optimization, safety engineering, testing, training, education, and video games.

In this study, a survey was conducted on the research of care for elderly people with dementia in the context of using ICT tools. Text-mining software was used to identify articles related to dementia care for elderly people and computer simulation. We then investigated potential opportunities to use computer simulation in education related to dementia care for elderly people.

\section{Objective}

The purpose of this study was to survey the use of ICT tools in educating caregivers of elderly people with dementia, with a focus on the use of computer simulation.

\section{Method}

We used two publicly available databases on the Internet: Igakuchuou zasshi and PubMed.

Igaku chuou zasshi (Ichushi): The Ichushi database is maintained by the non-profit Japan Medical Abstracts Society, and is one of the largest databases in Japan that includes Japanese medical and nursing articles. This database consists of about 5000 journals and posts 6,300,000 articles indexed using a thesaurus structure.

PubMed: PubMed comprises more than 22 million citations for biomedical literature from MEDLINE, life science journals, and online books. Some citations include links to full-text articles from PubMed Central and publisher web sites.

Thesaurus words in articles from Ichushi and PubMed were analyzed using the text-mining software "Trend Search”. This software was developed by FUJITSU and is commercially available [1]. The analysis provides a concept map of relational words, with the strength of the relation between words reflected in the line size and distance between them. For ethical purposes, articles were anonymized for analysis.

\section{Results and Considerations}

The literature search for article abstracts using the query phrases "elderly people with dementia AND simulation" identified four articles from Ichushi (2007-2010).

The most frequent terms identified in the analysis were "simulated patients (SP) $\rightarrow$ scale, teaching, participation, studying, case example, practice", which were associated with "understanding $\rightarrow$ feeling, training, elderly, students, subjective feeling, experience of older user" and "indication, learning, comunication, care”. Only four articles were identified, suggesting that little research has been conducted on this subject to date (Figure 1, Mapping of "Ichushi" made of remake by Trend search's results).

The literature search for "elderly people with dementia AND simulation" retrieved 16 abstracts from PubMed (1992-2012). The most freqent terms were "care”, “dementia”, and "of environment”, which were associated with "cognitive $\rightarrow$ individuals $\rightarrow$ is paper $\rightarrow$ incidence, prevalence, simulations" and "virtual $\rightarrow$ memory $\rightarrow$ episodic, patients". Consistent with the Ichishi search, these results also support the lack of research on this subject (Figure 2, Mapping of "Ichushi” made of remake by Trend search’s results).

Education using ICT tools is a relatively new concept in Japan. For example, Medicus Shuppan Publisher Co., Ltd. [2] is a Japanaese publishing house that specializes in educational tools for medicine and nursing. In addition to publishing books and magazines, Medicus sells computer software, such as Medicus DS. Medicus's battery of software (Figure 3) is a good example of commericial success of its kind in Japan. One educational program, "Raku-Raku Shindenzu Training DS" (Easy ECG Training DS), is composed of a "dictionary of keys to ECG reading” that explains ECG and arrhythmia reading in simple terms, and has graded (beginner, intermediate, 


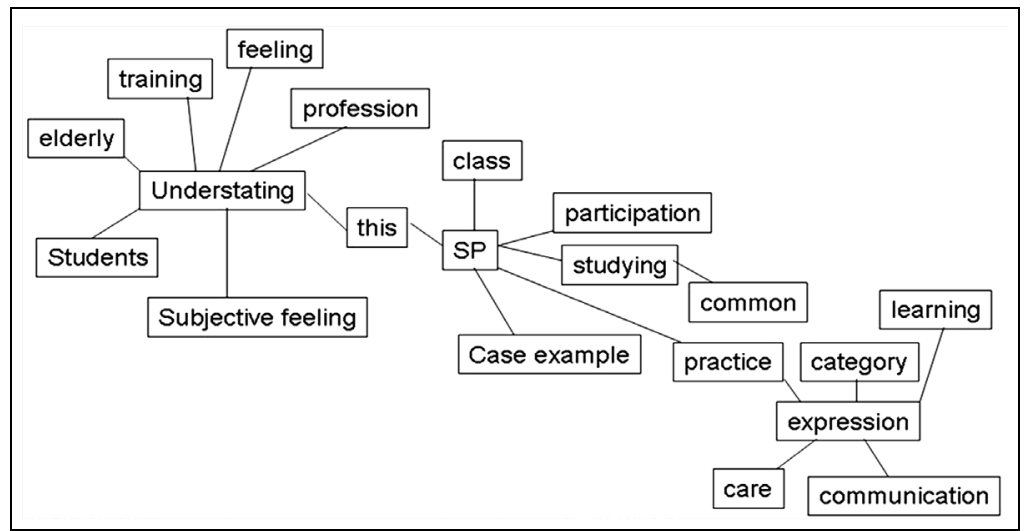

Figure 1. Mapping of “Ichushi” made of remake by Trend search’s results.

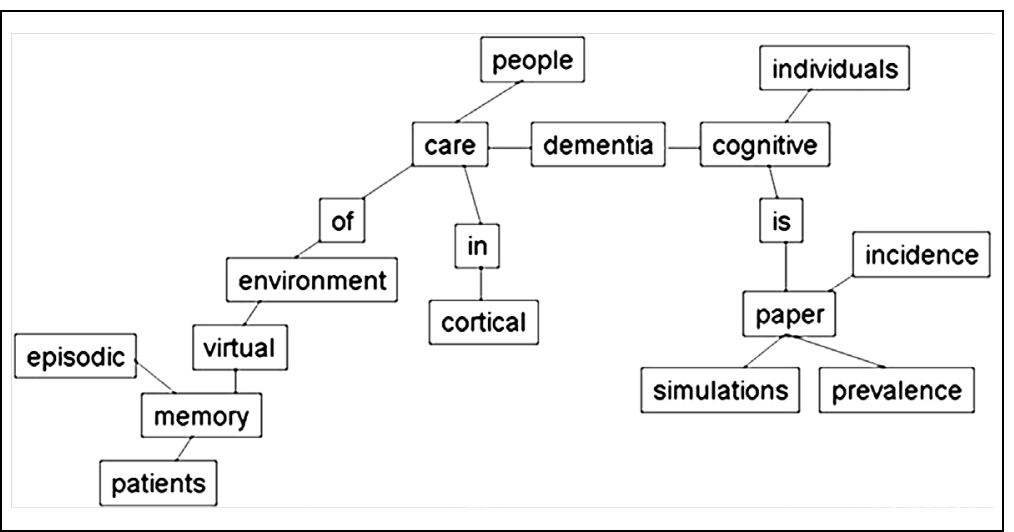

Figure 2. Mapping of “Pubmed” made of remake by Trend search’s results.
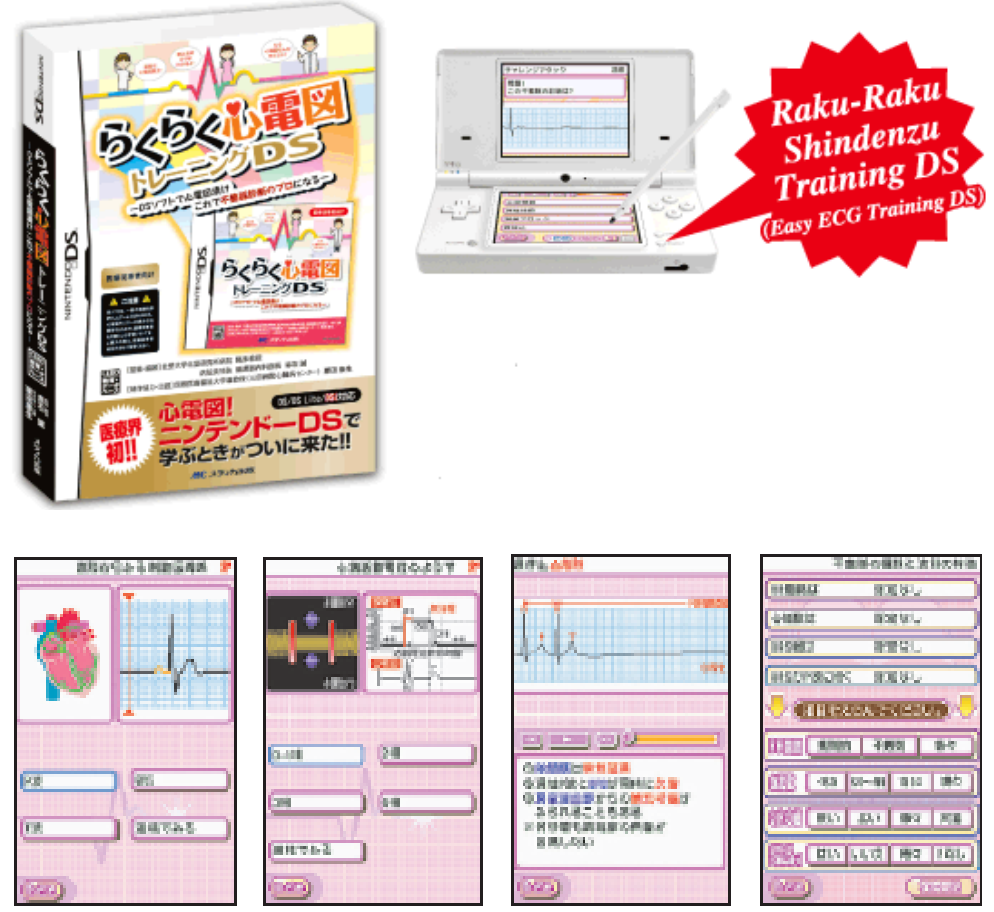

Figure 3. Example of e-learning software. 
and advanced) multiple-choice (four choices) questions called "Challenge Attack" to test ECG waveform reading ability.

Previous research has not fully addressed the use of computer simulation in the care of elderly people with dementia. We anticipate that opportunities to use simulation systems for dementia care education will increase in the future.

\section{Future Plans}

Our survey results revealed a lack of research on computer simulation training for dementia care for elderly people. One of the most effective ways to develop human resources in the field of dementia care is to develop a learning tool that efficiently provides the learner with sufficient knowledge regarding dementia. Software for dementia care would instruct users on how to communicate with elderly patients with dementia, and would also show the consequences of improper/poor communication. This can be achieved by developing virtual agents in the software that simulate a wide spectrum of elderly patients with dementia.

\section{References}

[1] Fujitsu (2012) Trend Search. http://software.ssri.co.jp/fuji/ts_proinfo.html

[2] Medicus Shuppan, Publisher Co., Ltd. http://www.medica.co.jp/ds/series/eng/index.php 